\title{
A single centre retrospective review of 25 patients treated for full arch edentulism with the All-on-4® concept
}

SADJ July 2019, Vol. 74 No. 6 p287 - p292

N Vafaei ${ }^{1}$, C Ferretti $^{2}$

\section{SUMMARY}

\section{Purpose}

To establish the outcomes and their reproducibility for the All-on-4® treatment protocol for full arch edentulism.

\section{Materials and Methods}

A retrospective observational cohort study of patients treated at a surgical private practice. Primary outcomes were three year cumulative implant and prosthesis survival. Secondary outcomes were surgical or prosthetic complications not resulting in implant or prosthesis loss. Marginal bone loss, measured on standardized panoramic radiographs was recorded if the loss exceeded Albrektsson's standards (less than $1 \mathrm{~mm}$ in the first year and maximum of $0.2 \mathrm{~mm}$ per year thereafter).

\section{Results}

Twenty-five patients (15 women, 10 men) were reviewed, with 124 implants supporting 31 full-arch fixed prostheses. Twenty had greater than three year follow-ups (range 40.7-139.7 months, mean 96.9 months), comprising 96 implants, and 26 jaws. Three implants failed in two patients, (survival rate 97.9\%). One prosthesis failed (96.2\% survival rate). Two patients required prosthesis replacement due to implant loss. One abutment screw fractured, which required replacement. A total of seven implants, in four different patients, had marginal bone loss in excess of the Albrektsson norm.

\section{Conclusion}

This study found that the reliability and predictability of the All-on-4® concept is verifiable for the treatment of total edentulism.

\section{Author affiliations:}

1. Nika Vafaei: BDS, MDent(MFOS), FCMFOS(SA), Private practice.

2. Carlo Ferretti: BDS, MDent (MFOS), FCD (SA) MFOS, Department of Maxillofacial and Oral Surgery, University of Pretoria, South Africa, Private Practice.

ORCID Number: 0000-0002-8862-391X

Corresponding author: Carlo Ferretti

P.O Box 75471, Gardenview 2047, South Africa. Email: ferretti@mweb. co.za. Tel: +27 (0)116159595

Author contributions:

1. Nika Vafaei: Principal researcher: Data collection $-70 \%$, Analysis $-80 \%$, Write-up - 60\%

2. Carlo Ferretti: Second author: Conception - 100\%, Surgery 100\%, Data collection: 20\%, Analysis: 30\%, Write-up: 40\%
Keywords

All-on-4®, implant rehabilitation, long-term survival.

\section{INTRODUCTION}

Edentulism is a common condition in elderly patients, and has a negative impact on oral health related quality of life. ${ }^{1}$ The goal of rehabilitation is to improve oro-dental function with a prosthesis that restores function and aesthetics with the lowest possible morbidity.

The loss of posterior dentition at an early age causes significant resorption of alveolar bone in the posterior segment of the jaws. Decreasing bone volume ultimately results in the surfacing of the inferior alveolar nerve in the mandible, and in sinus pneumatisation in the maxilla. This makes the placement of dental implants impossible, without reconstructive procedures such as grafting.

Classical implant treatment for edentulous patients with loss of the volume of posterior bone was initially described by Brånemark and colleagues in the 1970's. ${ }^{2}$ Four to six vertical and parallel implants are placed in the anterior mandible or maxilla, to support a cantilevered full-arch prosthesis. Whilst this treatment was and remains a viable option for restoring edentulous jaws, the reported complication rates would, by today's standards, be considered relatively high. ${ }^{2}$

It has been considered that these elevated complication rates may be due to excessive cantilever length. ${ }^{3-5}$ Cantilever lengths of less than $15 \mathrm{~mm}$ yield decreased marginal bone loss, resulted in higher implant survival, and decreased prosthesis failure as a consequence of better load distribution. ${ }^{3-6}$ To achieve the desired reduction in cantilever length, one may either shorten the dental arch or place the posterior implants more distally in the arch.

One method of enabling the distalizing of implants is to restore posterior bone volume with procedures such as bone grafting, sinus or ridge augmentation, and nerve lateralization. These approaches incur additional surgeries, higher costs, longer treatment time, and greater morbidity. An alternative implant placement philosophy introduced by Mattsson and colleagues and shortly thereafter by Krekmanov, exploited all usable bone anterior to the mental nerve in the mandible, and 
anterior to the maxillary sinus in the maxilla by placing the two distal implants at an angle, thus decreasing cantilever length.6,7 Subsequently Maló and colleagues modified this approach, reducing the number of implants to four per arch. ${ }^{8}$

The All-on-4® (NobelBiocare $₫, \quad K l o t e n, \quad S w i t z e r l a n d)$ design allows for the rehabilitation of an edentulous jaw by placing four implants in such a manner as to exploit as much of the available bone as possible, thus reducing the need for augmentation procedures. This is accomplished by placing two implants anteriorly (axially), and two posteriorly (tilted between $30^{\circ}$ and $45^{\circ}$ to the occlusal plane). ${ }^{8}$ By tilting implants posteriorly, longer implants can be placed which improves primary stability.

Increasing the antero-posterior spread of the implants decreases cantilever length and reduces the risk of prosthesis fracture due to the more favourable load distribution. ${ }^{6,7,9,10}$ In addition to the reduced surgical morbidity, this concept offers numerous biomechanical advantages which have been validated in finite element analysis studies. Inclined implants, in conjunction with a short cantilever, decrease stress on the peri-implant cortical bone, on the framework and implants.,11-14 Success rates using the All-on- $4 \AA$ technique compare favourably with traditional vertical implants. , $^{8,10,15,16}$

Since the early reports by Maló and colleagues, several other authors have reported high cumulative implant

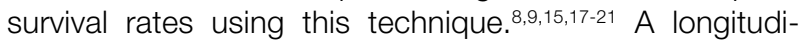
nal study by Maló and colleagues including 245 patients with completely edentulous mandibles rehabilitated with the All-on- $4 \AA$ treatment concept, reported cumulative implant survival rates (depending on follow-up period) ranging from $93.8 \%$ at $6-10$ year follow-up, to $97.6 \%$ at a one year follow-up. 22

Another study by the same group reported the results for 968 implants placed in 242 patients in the maxilla and the mandible. A cumulative implant survival rate of $98 \%$ was obtained at a five year follow-up. ${ }^{23}$ Duello summarized the findings of these and other authors in a literature review, and reported that the implant survival rate was influenced by length of follow-up. In the maxilla, short term follow-up yielded up to $100 \%$ implant survival, and decreased to $92.5 \%$ after seven years. Similarly, mandibular implants with short term follow-up had up to $100 \%$ survival rate, dropping to $93.8 \%$ at a 10 year follow-up..$^{24,25}$

With regard to marginal bone loss, the consensus is that there is no significant difference between traditional vertical splinted implants and a combination of axial and tilted implants. ${ }^{6,10,16,26-29}$ Therefore this technique is a good alternative for clinicians treating edentulous patients requiring full-arch rehabilitation, in particular those with atrophic jaws that would normally require traditional bone grafting before implant placement.

The purpose of this study is to present our experiences with the All-on- $4 \otimes$ technique for treatment of full jaw edentulism from a single surgical practice using only clinical and radiographic data garnered in the process of treatment. No other investigations were done for research purposes. It is the intention to assess the outcomes for this treatment protocol in a general oral surgical private practice. The accumulation of reproducible results using the All-on-4® technique from a variety of sources, particularly after an above average follow-up period, aids in providing further scientific data to support this treatment rationale.

\section{METHODS}

A retrospective cohort observational study of patients was performed at a single surgical private practice. Patients were either edentulous or had a dentition deemed unrestorable by the attending clinician.

Pre-treatment assessment included clinical examination, panoramic radiography, prosthodontic and surgical consultation, and when indicated, cone beam computational tomography. Patients were offered treatment with this protocol if the alveolar bone volume was insufficient for traditional implant placement of six fixtures per jaw, and if they met the following criteria:

- good general health with acceptable oral hygiene.

- an alveolus of at least $10 \mathrm{~mm}$ height.

- for immediate loading, implants achieved an insertion torque of at least $35 \mathrm{Ncm}$.

- in the mandible, the position of the mental nerve allowed for distalization of the prosthetic platform (mental foramen was at least $5 \mathrm{~mm}$ below alveolar crest).

- anterior maxillary wall position allowing for placement of tilted implants where the prosthetic interface would emerge at least at the position of the upper first premolar.

The following data were recorded: demographic data, number of jaws treated, whether the implants were placed in edentulous jaws or immediately following extraction of non-salvageable teeth (immediate placement), insertion torques, whether a prosthesis was placed immediately (immediate loading no later than 48 hours post placement), or whether a delayed loading protocol was used (no earlier than three months). Follow up appointments assessed prosthesis integrity, gingival health and radiographic bone levels. Patient identification was removed from the data set to maintain anonymity.

\section{Surgical protocol}

The alveolus was exposed using a crestal incision with distal relief. In the maxilla, the nasal rim and anterior lateral maxillary wall were identified, and in the mandible the mental foramen was identified. Implant placement was guided by the All-on- $4 \AA$ surgical guide The guide was placed into a $2 \mathrm{~mm}$ osteotomy made in the midline of the jaw and the metal band was contoured to the arch form (Figure 1).

The anterior maxillary wall was identified and explored through a window in the lateral maxillary sinus wall and the tilted implant placed just anterior to that (Figure 2). In the mandible, with the guide in position, preparation of the osteotomy sites for the tilted implants was commenced on the alveolar crest above the distal aspect of the mental foramen (Figure 3). 


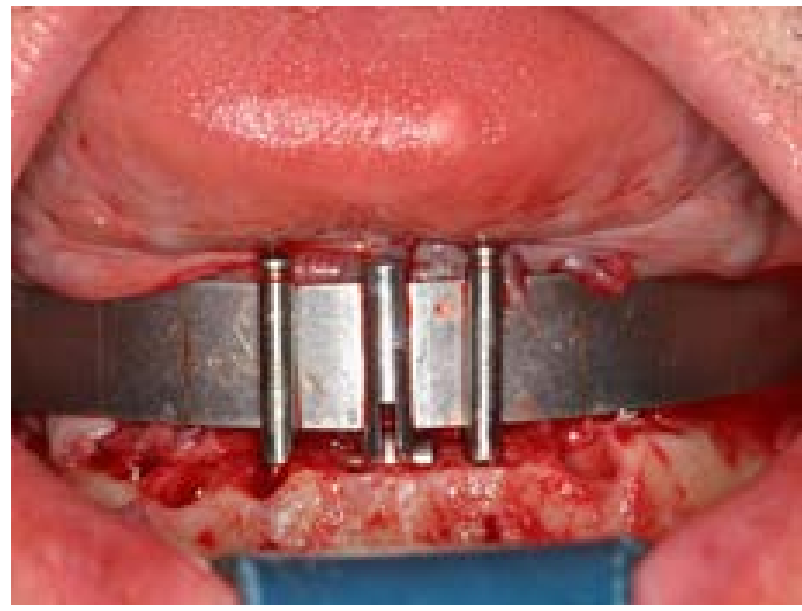

Figure 1. All-on-4® surgical guide in the mandible with the metal band contoured to the arch form facilitating optimal spacing, parallelism and inclination of the implants.

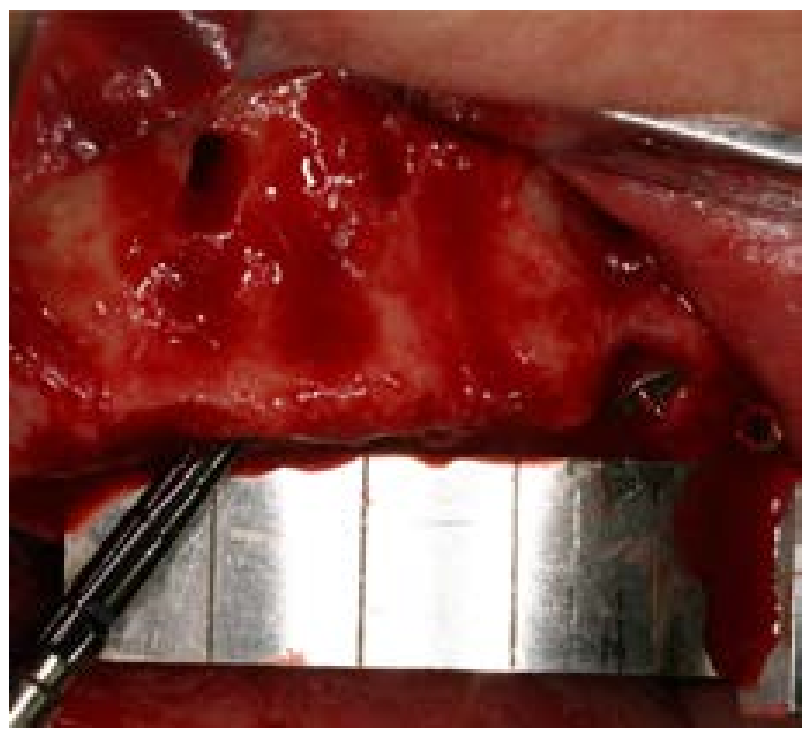

Figure 2. The anterior wall of the maxillary sinus is identified through a window in the lateral wall. The tilted implant is placed just anterior to the anterior maxillary sinus wall. Angulation of the drill is guided by markings on the guide.

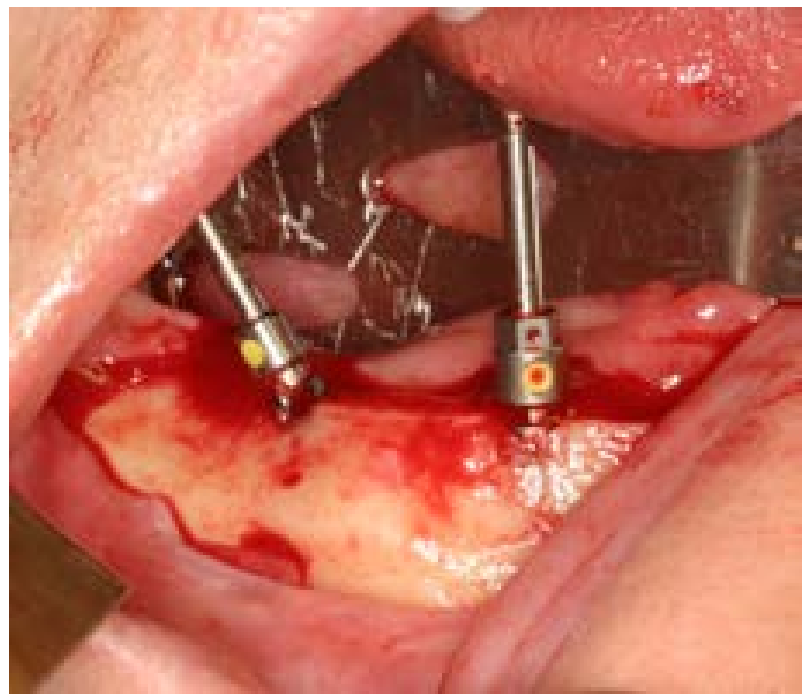

Figure 3. Preparation of the osteotomy site in the mandible for placement of tilted implants above the distal aspect of the mental foramen.

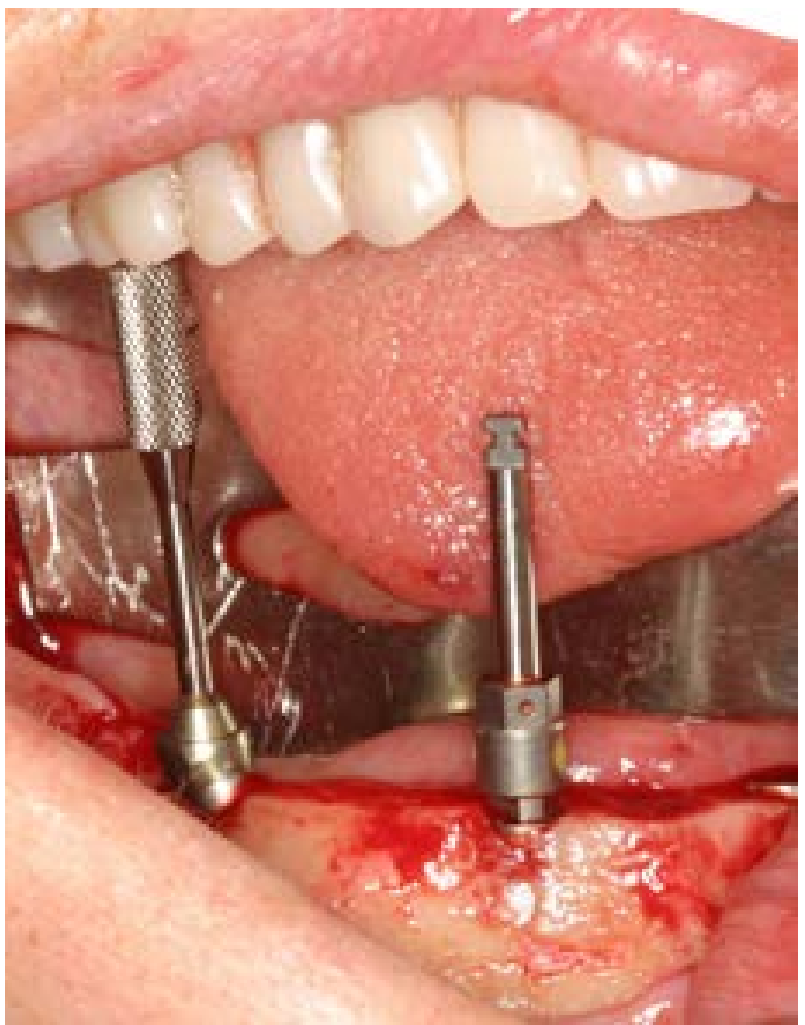

Figure 4. A trial prosthetic abutment in situ used to ensure correct rotational position of prosthetic platform.
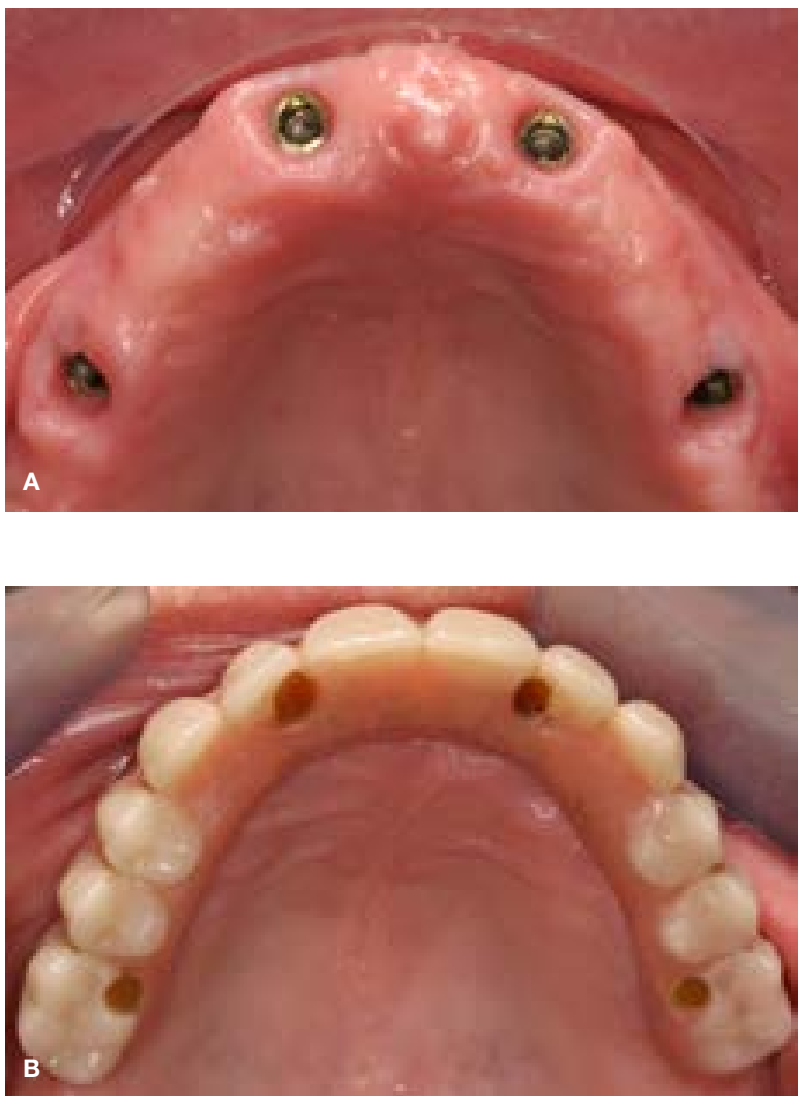

Figure 5. Occlusal view of maxilla (A), and with prosthesis in situ (B) showing favourable implant distribution. 
NobelReplace Tapered Groovy ${ }^{\circledR}$ implants were placed in all patients. A trial prosthetic abutment was used to ensure the correct rotational position of the prosthetic platform, and to determine whether a 17 or 30 degree multi-unit abutment would be required (Figure 4). If prosthodontic requirements dictated, straight abutments were placed in the axial implants.

Torque out values were recorded as fair (20-29 Ncm), good $(30-39 \mathrm{Ncm})$ and excellent $(>40 \mathrm{Ncm})$. Patients were offered immediately loaded prostheses if implant stability met the minimum torque out value of $35 \mathrm{Ncm}$, and was contingent on the patients' budget (thus not all patients whose implants could have been loaded immediately actually accepted this option). Alternatively, conventional dentures were used as a temporary measure.

The canon for the classification of an implant post-placement is that described by Albrektsson et al. An implant is classified as successful when it meets all four of the following criteria: ${ }^{30}$

1. No radiolucency around the implant.

2. No signs of infection, pain or ongoing pathological processes present at the implant site.

3. The implant is restored and functionally loaded.

4. The prosthesis is stable for multiple implants supporting a complete arch prosthesis.

An implant is classified as "surviving" when it remains in the jaw and is functionally loaded even though all the individual success criteria may not be fulfilled; and as a "failed" implant if the implant has fractured beyond repair or cannot be classified as successful or surviving. ${ }^{30}$

In this study implant success and survival rates were calculated only for patients for whom there was three year or greater follow-up. A prosthesis was classified as surviving if the original prosthesis remained in situ and the patient was content with its function. More detailed assessment of prosthesis success is beyond the scope of our expertise and was thus not considered. A prosthesis requiring replacement, unrelated to implant loss, was deemed a primary prosthesis failure. Prosthesis loss due to implant failure was considered a secondary prosthesis failure. Loss or fracture of a component of a prosthesis was deemed a minor prosthetic complication if it did not require replacement of the whole prosthesis.

Marginal bone loss was measured on orthopantograph films. All radiographs were taken on the same machine (Sirona Orthophos, Sirona Dental Systems, Bensheim, Germany) at standardized exposure settings and with standardized patient positioning.

Calibration of the radiograph was done by measuring the radiographic length of an implant and dividing it by the actual implant length to obtain a magnification factor. This was then used to adjust the bone loss measurement obtained from the radiograph.

The implant prosthetic platform was used as the fixed reference for measurement and bone loss was measured to the depth of the peri-implant defect. The benchmark for the assessment of peri-implant bone loss is the peri-apical radiograph. This had not been obtained on a routine basis on all implants at regular intervals and therefore we elected to not use that data. Albrektsson et al. described the maximum acceptable bone loss commensurate with implant success. The criteria are: bone loss less than $1 \mathrm{~mm}$ in the first year and thereafter $0.2 \mathrm{~mm}$ per year. ${ }^{30}$ Bone loss was recorded only for those implants that exceeded the criteria of Albrektsson et al. ${ }^{30}$

\section{RESULTS}

Twenty-five patients were reviewed in the study, 10 males and 15 females, with a mean age of $61 \pm 9.58$ years (range of 37-74). Total number of implants placed was 124 , 56 in the maxilla and 68 in the mandible. There were 31 jaws: in nine patients maxilla only, in 10 patients mandible only, and in six patients both maxilla and mandible. Of these 31 jaws, 26 had received immediate implant placement whilst the remaining five jaws were edentulous at the time of implant placement. In 19 jaws, loading occurred after implant integration (delayed), whilst in 12 jaws immediate loading was done.

\section{Torque values}

One hundred and eighteen implants (95.2\%) had peak torque values greater than $40 \mathrm{Ncm}$ (excellent stability), four implants (3.2\%) had good primary stability, and 2 (1.6\%) had fair primary stability.

\section{Follow-up}

Two patients passed away, with their implants and prostheses surviving at the last follow-ups for, respectively, six months and 18 months post treatment. Three patients had follow-ups of less than three years and were therefore excluded from the calculation of the results. The follow-up for the remaining 20 patients included in this cohort ranged from 40.7-139.7 months, with a mean of $96.9 \pm 20.4$ months

\section{Implant failure}

Three implants failed in two patients. In one patient two implants failed 10 years post placement (one tilted and one axial implant). This patient's implants had been immediate placements without immediate loading. In the other patient, a single tilted implant (immediate placement with immediate loading) failed two years post placement and the patient was subsequently lost to follow-up. The 20 patients who had greater than three year follow-ups received 96 of the 124 implants placed. In this cohort, 87 of the 96 implants were classified as successful (90.6\%), the implant survival rate was $97.9 \%$ (94 of 96 implants).

\section{Marginal bone loss}

Marginal bone loss exceeded the generally accepted standard in seven implants in four patients. Three implants in one patient, whose prosthesis remained in situ after 12 years, had marginal bone loss of $6.8 \mathrm{~mm}$, $5.6 \mathrm{~mm}$ and $4.4 \mathrm{~mm}$ in two axial and one tilted mandibular implants, respectively. The second patient whose prosthesis had been in situ for seven years had periimplantitis and marginal bone loss of $7.8 \mathrm{~mm}$ around a single axial implant in the maxilla. The third patient had marginal bone loss around a single axial implant in the mandible measuring $7.5 \mathrm{~mm}$ after 8 years with the 
prosthesis in situ. The fourth patient had marginal bone loss around the two axial mandibular implants, measuring $5.3 \mathrm{~mm}$ and $4.8 \mathrm{~mm}$. In two of these patients, a single implant required debridement under local anaesthetic. The remaining patients were referred back to the restoring practitioners to remove the prosthesis and to undergo debridement of the periimplant space. They were enrolled in a high supervision oral hygiene maintenance programme.

\section{Prosthesis failure}

Treatment with this surgical design allowed for a broad distribution of implant prosthetic platforms and the manufacture of a prosthesis with 10-12 teeth but with very short cantilevers (Figures 5A \& B). One patient had primary prosthesis failure and two patients had secondary prosthesis failure. One patient had a fractured prosthetic screw in an angled abutment requiring replacement of the abutment. One of the 20 patients had a primary prosthesis failure (1 of 26 jaws) due to fracture, resulting in a $96.2 \%$ prosthesis survival rate.

\section{Surgical complications}

The only meaningful surgical complication was lower lip paraesthesia experienced by two patients, which resolved with no active treatment after four and five weeks respectively.

\section{DISCUSSION}

This study evaluated the outcomes of the All-on-4® treatment concept for rehabilitation of edentulous jaws in a surgical private practice. The aim was to determine results and to compare them with those obtained by others. Duplication of results is an important mechanism for strengthening the scientific validity of a treatment philosophy.

Although the criteria enunciated by Albrektsson et al. ${ }^{30}$ are widely accepted as the standard for differentiating implant success, survival and failure, the terms implant success and implant survival are often used inappropriately and interchangeably. Careful scrutiny of the studies we reviewed (and to which we are comparing our results), reveal that in fact, the most common criterion used was that of implant survival. ${ }^{14-18,22,23,31-33}$ Thus for the purpose of comparison with other studies, it is possible to use only the implant survival rate. In this study, the cumulative implant survival rate (at a mean follow-up of 96.9 months) for this cohort is $97.9 \%$, and the prosthesis survival rate is $96.2 \%$.

A recent systematic review of 24 studies summarized the clinical outcomes of the All-on-4® technique. The pooled mean follow-up period for the studies under review was five years (range 3-10 years). ${ }^{33}$ The implant survival rate was $99.8 \%$ at a two year follow-up, with a decline to $95 \%$ after five years. ${ }^{33}$ Two of the studies reviewed had follow-ups of up to 10 years. Maló et al. reported cumulative implant survival rates between $94.8 \%$ and $98.1 \%$ at five year follow-up, and 93.8$94.8 \%$ at 10 years. The mean follow-up in this study was six years, with only a small group of eight patients having a follow-up of 10 years. ${ }^{22}$ A follow-up study by the same authors in 2015 reported a cumulative implant survival rate of $95.4 \%$ at seven years for 260 of 324 patients. ${ }^{14}$ Lopes et al. reported a cumulative implant survival rate of $96.6 \%$ at a five year follow-up, in 23 patients. ${ }^{31}$ The results in these reports show cumulative implant survival rates between 96-98.4\% at a five year followup, with a decrease to 93.8-95.4\% survival rate after 7-10 years. ${ }^{10,14,22,31,32}$

Although the sample size is smaller than many of the above mentioned studies, the average follow-up in the current study was eight years, with nine of the 20 patients having a longer follow-up. Our implant survival rate of $97.9 \%$ at a mean follow-up of eight years is slightly higher than that obtained by other authors; however it is likely that a larger sample size would have yielded figures consistent with previous reports. 1,14,22,23,31,34

Peri-implant bone loss is an ongoing concern of much of implant research. A systematic review of complications associated with implant-supported fixed dental prostheses reported a peri-implant bone loss greater than $2 \mathrm{~mm}$ of $20.1 \%$ after five years and $40.3 \%$ after 10 years. ${ }^{35}$ A limitation of this study is the technique used for the measurement of marginal bone loss. It should be borne in mind that the measurement of peri-implant bone loss was not a primary objective of this study.

The reality of performing retrospective reviews of patients not previously recruited into a formal study (thus not subjected to investigations required only for research purposes, but only for clinical need) means that suboptimal data may be collected. The implications are that whilst the absolute figures for bone loss may be inaccurate, more importantly, the identification of implants that have had no bone loss as opposed to those that have, can be done very reliably with a screening panoramic radiograph. It has been contended that the use of any one parameter, including peri-implant bone loss, cannot be used as a determinant of success in implant treatment. ${ }^{36}$

The results of this study support the hypothesis that the rehabilitation of the edentulous mandible and maxilla using the All-on- $4 \AA$ concept is predictable and reliable. ${ }^{22}$ The numerous benefits of this technique are somewhat mitigated by the tenuous implant support offered by the implants for the prosthesis. The loss of a tilted implant invariably entails the loss of the prosthesis. This is one of the major disadvantages of the treatment concept over the traditional axial philosophy or the placement of more implants. Nevertheless, it is a complication that remains rare enough to not dissuade the clinician to consider this treatment option.

\section{CONCLUSION}

Our experiences with the All-on-4® technique have corroborated the findings of others. Within the limitation of this study, our results indicate that it is a treatment modality that is safe and predictable with a high implant survival rate even after up to 12 year follow-up.

\section{Acknowledgements}

We would like to thank Dr. Emil Cahi and Dr. Selwyn Kabrun for prosthodontic support, and Dr. Shailen Dullabh for data collection. 


\section{References}

1. Babbush C, Kutsko G, Brokloff J. The All-on-four immediate function concept with NobelActive Implants: a retrospective study. J Oral Implantol. 2011; 28(4) : 431- 45.

2. Brånemark PI, Hannsson BO, Adell R, et al. Osseointegrated implants in treatment of the edentulous jaw. Experience from a 10-year period. Scand J Plast Reconstr Surg Suppl. 1977; 12:1-132.

3. Shackleton JL, Carr L, Slabbert JCG, Becker PJ. Survival of fixed implant-supported prostheses related to cantilever lengths. J Prosthet Dent. 1994; 71:23-6.

4. Naert I, Quirynen M, Van Steenberghe D, Darius P. A study of 589 consecutive implants supporting complete fixed prostheses. Part II: prosthetic aspects. J Prosthet Dent. 1992; 68:949- 56.

5. Rangert B, Jemt T, Jomeus L. Forces and moments on Brånemark implants. Int J Oral Maxillofac Implants 1989; 4:241-7.

6. Mattsson T, Konsell P, Gynther G, et al. Implant treatment without bone grafting in severely resorbed edentulous maxillae. J Oral Maxillofac Surg. 1999; 57:281-7.

7. Krekmanov L, Kahn M, Rangert B, Lindström H. Tilting of posterior mandibular and maxillary implants for improved prosthesis support. Int J Oral Maxillofac Implants 2000; $15: 405-14$

8. Maló P, Rangert B, Nobre M. All-on-4 immediate function concept with Brånemark system implants for completely edentulous mandibles: a retrospective clinical study. Clin Implant Dent Relat Res. 2003; 5:S2 - S9.

9. Maló P, de Araujo Nobre M, Lopes A. The use of computer-guided flapless implant surgery and four implants placed in immediate function to support a fixed denture: preliminary results after a mean follow-up period of thirteen months. J Prosthet Dent. 2008; 97(6 Suppl):S26-34, 2007. Erratum in: J Prosthet Dent. Mar; 99(3):167.

10. Aparicio, C, Perales, P \& Rangert, B. Tilted implants as an alternative to maxillary sinus grafting: a clinical, radiologic, and periotest study. Clin Implant Dent Relat Res. 2001; $3: 39-49$.

11. Zampelis A, Rangert B, Heijl L. Tilting of splinted implants for improved prosthodontic support: a two dimensional finite element analysis. J Prosthet Dent. 2007; 97: S255- S264.

12. Takahashi T, Shimamura I, Sakurai K. Influence of number and inclination angle of implants on stress distribution in mandibular cortical bone with All-on-4 Concept. J Prosthodont Res. 2010; 54:179-84.

13. Fazi G, Tellini S, Vangi D, Branchi R. Three-dimensional finite element analysis of different implant configurations for a mandibular fixed prosthesis. Int $\mathrm{J}$ Oral Maxillofac Implants. 2011; 26:752-9.

14. Maló P, De Araujo Nobre M, Lopes A, Ferro A, Gravito I. All-on-4 Treatment Concept for the rehabilitation of the completely edentulous mandible: A 7-year clinical and 5-year radiographic retrospective case series with risk assessment for implant failure and marginal bone level. Clin Implant Dent Relat Res. 2015; 17(2):531-41.

15. Maló P, Rangert B, Nobre M. All-on-4 immediate-function concept with Brånemark System implants for completely edentulous maxillae: a one-year retrospective clinical study. Clin Implant Dent Relat Res. 2005; 7(Suppl 1) : S88 - 94.

16. Calandriello R, Tomatis, M. Simplified treatment of the atrophic posterior maxilla via immediate/early function and tilted implants: a prospective one-year clinical study. Clin Implant Dent Relat Res. 2005; 7(Suppl 1):S1-12.

17. Maló P, Rangert B, Nobre M. "All-on-Four" immediate-function concept with Brånemark System implants for completely edentulous mandibles: a retrospective clinical study. Clin Implant Dent Relat Res. 2003; 5(Suppl 1) : 2 - 9.

18. Maló P, Lopez I, Nobre M. The All on Four concept. In: Babbush C, Hahn J, Krauser J, Rosenlicht J. Dental Implants The Art and Science. $2^{\text {nd }}$ edition. Missouri, Saunders Elsevier Inc, 2010:435.
19. Weinstein R, Agliardi E, Fabbro MD, Romeo D, Francetti L. Immediate rehabilitation of the extremely atrophic mandible with fixed full-prosthesis supported by four implants. Clin Implant Dent Relat Res. 2012; 14(3):434-41.

20. Pomares C. A retrospective clinical study of edentulous patients rehabilitated according to the 'all on four' or the 'all on six' immediate function concept. Eur J Oral Implantol. 2009; 2(1): 55-60.

21. Testori T, Del Fabbro M, Capelli M, Zuffetti F, Francetti L, Weinstein RL. Immediate occlusal loading and tilted implants for the rehabilitation of the atrophic edentulous maxilla: One-year interim results of a multicenter prospective study. Clin Oral Implants Res. 2008; 19(3):227-32.

22. Maló $P$, De Araujo Nobre M, Lopes A, et al. A longitudinal study of the survival of All-on-4 implants in the mandible with up to 10 years of follow-up. J Am Dent Assoc. 2011; $142: 310-20$.

23. Maló P, De Araujo Nobre M, Lopes A, et al. "All-on-4" immediate function concept for completely edentulous maxillae: a clinical report on the medium (three years) and long-term (five years) outcomes. Clin Implant Dent Relat Res. 2011; 14(Suppl 1) : e139-50.

24. Duello GV. An evidence-based protocol for immediate rehabilitation of the edentulous patient. J Evid Based Dent Prac. 2012; 12(3 Supp) :172-81.

25. Patzelt SB, Bahat O, Reynolds MA, et al. The All-on-Four treatment concept: a systemic review. Clin Implant Dent Relat Res. 2013. http://dx.doi.org/10.1111/ CID.12068.

26. Bellini CM, Romeo D, Galbusera F, Taschieri S, Raimondi MT, Zampelis A, Francetti L. Comparison of tilted versus non tilted implant-supported prosthetic designs for the restoration of the edentulous mandible: a biomechanical study. Int J Oral Maxillofac Implants 2009; 24(3):511-7.

27. Del Fabbro M, Bellini CM, Romeo D, Francetti L. Tilted implants for the rehabilitation of edentulous jaws: a systematic review. Clin Implant Dent Relat Res. 2010; 14(4):612-21.

28. Ata-Ali J, Penarrocha-Oltra D, Candel-Marti E, PenarrochaDiago M. Oral rehabilitation with tilted dental implants: a meta-analysis. Med Oral Patol Oral Cir Bucal. 2012; 17:e582-e587.

29. Monje A, Chan HL, Suarez F, Galindo-Moreno P, Wang HL. Marginal bone loss around tilted implants in comparison to straight implants: a meta-analysis. Int J Oral Maxillofac Implants 2012; 27:1576-83.

30. Albrektsson T, Zarb G, Worthington P, Eriksson AR. The long-term efficacy of currently used dental implants: a review and proposed criteria of success. Int J Oral Maxillofac Implants. 1986; 1:11-25.

31. Lopes A, Maló, P, de Araújo Nobre, M. and SanchezFernández, E. The NobelGuide $®$ All-on-4® Treatment Concept for rehabilitation of edentulous jaws: a prospective report on medium- and long-term outcomes. Clin Implant Dent Relat Res. 2015; 17: e406-e416.

32. Balshi TJ, Wolfinger GJ, Slauch RW, Balshi SF. A retrospective analysis of 800 Brånemark System implants following the All-on-FourTM Protocol. J Prosthodont. 2013; 23:83-8.

33. Soto-Peñaloza D, Zaragozí-Alonso R, Peñarrocha-Diago M Peñarrocha-Diago M. The all-on-four treatment concept: Systematic review. J Clin Exp Dent. 2017; 9(3): e474-88.

34. Chan $\mathrm{MH}$, Holmes C. Contemporary all-on-4 concept. Dent Clin N Am. 2015; 59:421-70.

35. Papaspyridakos P, Chen CJ, Chuang SK, Weber HP, Gallucci GO. A systematic review of biologic and technical complications with fixed implant rehabilitations for edentulous patients. Int J Oral Maxillofac Implants 2012; 27(1):102-10.

36. Papaspyridakos P, Chen CJ, Singh M, Weber HP, Gallucci GO. Success criteria in implant dentistry: a systematic review. J Dent Res. 2012; 91(3): 242-8. 